\title{
Misurare il contributo della comunicazione alla corporate reputation per la creazione di valore*
}

\author{
MARia Teresa CuOMO** Debora TORTORA*** \\ GERARDINO METALLO****
}

\begin{abstract}
Obiettivo del paper: Il lavoro si propone di analizzare la correlazione tra il livello di reputazione delle organizzazioni e la sua "rilevanza", in termini di comunicazione, quale "strumento" per migliorare le performance aziendali.

Metodologia: E stata realizzata una ricerca esplorativa su un gruppo di imprese del settore alberghiero con sede in provincia di Salerno, per valutare se e come il loro quoziente reputazionale, calcolato rifacendosi ad un originale modello multistadio, possa essere influenzato dall'attività di comunicazione.

Risultati: Il progetto di ricerca fornisce un contributo rilevante alle problematiche di governance delle imprese, dal momento che la comunicazione qualifica interdipendenze sistemiche sia da un punto di vista interno che esterno e, allo stesso tempo, suggerisce possibili influenze sulla creazione di valore, sintetizzate nel reputation quotient, da parte dell'impresa.

Limiti della ricerca: Lo studio si basa su una esemplificazione, utilizzando un panel di aziende nel settore alberghiero. Sarebbe utile poter allargare la base campionaria ed i settori economici analizzati.

Implicazioni pratiche: La comunicazione assume un ruolo strategico per la diffusione dei risultati conseguiti in termini di creazione di valore, a patto che si basi su performance consolidate. Al contrario, il valore si disperde quando gli investimenti in comunicazione sono finalizzati a mascherare prestazioni mediocri, potendo, in casi estremi, acuire o addirittura determinare situazioni di crisi aziendale, violando "il patto di trasparenza" stipulato dall'impresa con i propri stakeholder.
\end{abstract}

* Pur essendo frutto di comune elaborazione, il lavoro va attribuito per il par. 1 a Gerardino Metallo, per il par. 2 a Maria Teresa Cuomo, per il par. 3 a Debora Tortora, mentre i par. 4 e 5 sono stati curati congiuntamente dagli Autori.

** Associato di Economia e Gestione delle Imprese - Università degli Studi di Salerno email: mcuomo@unisa.it

*** Assegnista di ricerca di Economia e Gestione delle Imprese - Università degli Studi di Salerno

email: dtortora@unisa.it

**** Ordinario di Economia e Gestione delle Imprese - Università degli Studi di Salerno email:gemetall@unisa.it 
Originalità del lavoro: Utilizzando lo Schema delle Aree $R Q$ si può verificare se nel governo d'impresa sia stato attribuito un ruolo "adeguato" alla comunicazione quale vettore per la creazione di valore.

Parole chiave: quoziente reputazionale; modello delle aree reputazionali; investimenti in comunicazione

Purpose of the paper: The research paper aims to analyze the correlation between the reputation level and its "prominence", in terms of communication, as a "tool" to improve firm performance in a competitive context.

Methodology: In order to validate the research hypotheses, an exploratory qualitative research has been put in place on a cluster of smes based in province of Salerno to ascertain if and how their reputation quotient can be influenced by communication. The reputation quotient is based on a model of multilevel reputation measurement.

Findings: The research project constitutes a relevant contribution to management and governance issues, seeing as communication qualifies systemic interdependence from both an internal and external perspective and, at the same time, suggests first hand evidence of the variability of firm value creation, in terms of reputation quotient.

Research limits: The study is based on an example, using a panel of smes in the hospitality sector. It would be useful to enlarge the base of the sample and the economic sectors.

Practical implications: Communication acquires a strategic role for the expanding of results achieved for the purpose of creating value, provided it is based on consolidated performance. On the contrary, value is dispersed when investments in communication are aimed at concealing mediocre performance, which can even determine firm crises, in terms of "breaching the pact of transparency" stipulated by the firm with its stakeholders.

Originality of the paper: Using the RQ Areas Scheme it can be verified if - in corporate governance - a "suitable" role could be attributed to communication as a vector of value creation.

Key words: reputation quotient; $R Q$ Areas Scheme; communication investments

\section{Premessa}

In quanto istituzioni sociali a finalità plurime, le organizzazioni imprenditoriali si pongono come obiettivo prioritario la creazione di valore allargato, attraverso l'impiego di un complesso ed eterogeneo stock di fattori, detenuti sotto forma di possesso e/o di controllo. Tra essi, un ruolo preminente va riconosciuto a quegli aspetti legati agli ambiti della conoscenza nell'impresa, come insieme di dati, rapporti, indicazioni che alimentano le relazioni sviluppate all'interno dell'organizzazione (presidio delle attività di comunicazione interna); altro aspetto rilevante è, poi, la conoscenza sull'impresa, riferendosi alle informazioni che vengono diffuse nell'ambito delle relazioni che si sviluppano tra questa ed un'ampia gamma di pubblici esterni, da cui traggono origine l'immagine e, conseguentemente, 
la credibilità e la reputazione aziendale nell'ambiente - mercato di riferimento (area della comunicazione esterna) ${ }^{1}$.

Invero, per il successo delle imprese proprio la reputazione, quale giudizio diffuso e sedimentato nel tempo che $i$ diversi interlocutori danno della credibilità delle sue affermazioni, della qualità e affidabilità dei suoi prodotti e della responsabilità delle sue azioni, trova ampio riscontro in letteratura ${ }^{2}$, recuperando in principio sostegno in diverse teorie microeconomiche, come la Teoria dell'Agenzia (Fombrun e Shanley, 1990; Mutti, 2007) e la Teoria dei Giochi (Milgrom e Robert, 1982); queste ultime si focalizzano su una accezione di reputazione quale particolare attributo dell'organizzazione per il governo delle relazioni tra imprese e pubblici di riferimento (visione statica), non riuscendo a fornire giustificazione di un legame diretto tra corporate reputation e generazione di valore economico. Viceversa, altri studiosi hanno mostrato crescente considerazione per una interpretazione della reputazione d'impresa quale fenomeno dinamico, di cui interessa cogliere antecedenti formativi ed esiti raggiungibili. Pertanto, l'attenzione è stata posta alternativamente sul contributo dei framework riconducibili alla Insitutional Theory, focalizzata sull'incremento del consenso dell'organizzazione all'interno di contesti istituzionali, piuttosto che sull'interpretazione delle performance quali segnali per la formazione delle percezioni degli stakeholder (Signaling Theory); infine, per una analisi degli "esiti" raggiungibili attraverso la gestione della reputazione aziendale può farsi riferimento al contributo della Resource-Based View, che vi riconosce un ruolo di asset strategico intangibile, in grado di generare rendite e vantaggi competitivi (Roberts e Dowling, 2002), incorporando una garanzia di difficile imitabilità dell'agire organizzativo (figura 1 ).

Le possibilità di sopravvivenza e di successo dell'impresa dipendono, quindi, dal circolo virtuoso generato tra l'accrescimento del patrimonio di risorse immateriali di conoscenza, fiducia e reputazione ${ }^{3}$ - non imitabile e, quindi, strategico (Barney,

1 In sostanza, muta l'ottica di analisi della creazione di valore, che si sposta dalla singola impresa al network nel quale essa si inserisce; da ciò, il valore creato non deriva esclusivamente dall'efficiente/efficace gestione delle funzioni aziendali, ma si propone come sintesi delle capacità relazionali tra organizzazioni e tra queste e pubblici, nell'ottica collaborativa della condivisione delle risorse: in primis, la conoscenza, reale motore e generatore di valore allargato. Nel presente lavoro, dunque, l'attenzione è concentrata sul ruolo della "conoscenza sull'impresa" ed in particolare, sulla "reputazione", per la creazione di valore.

2 In tema di generazione di valore, negli anni Settanta si affermano i primi studi di Corporate Reputation, con riferimento all'analisi dei processi decisionali d'impresa condotti in situazioni di incompleta o imperfetta informazione, che "forces external observers to rely on proxies to describe the preferences of rivals and their likely courses of action" (Fombrum e Van Riel, 1997, p. 6).

3 Infatti, "The spread of improper conduct among the managers of important companies negatively affects the reputation of the business environment in general, leading to growing levels of lack of confidence in relations. The cases of Parmalat and Enron are particularly significant in this respect" (Baccarani, 2008, p. 160). 
1991) - l'ampliamento del suo divario con i competitor ed il conseguente rafforzamento delle fonti del vantaggio strategico ${ }^{4}$.

Fig. 1: Pre-action to post-action

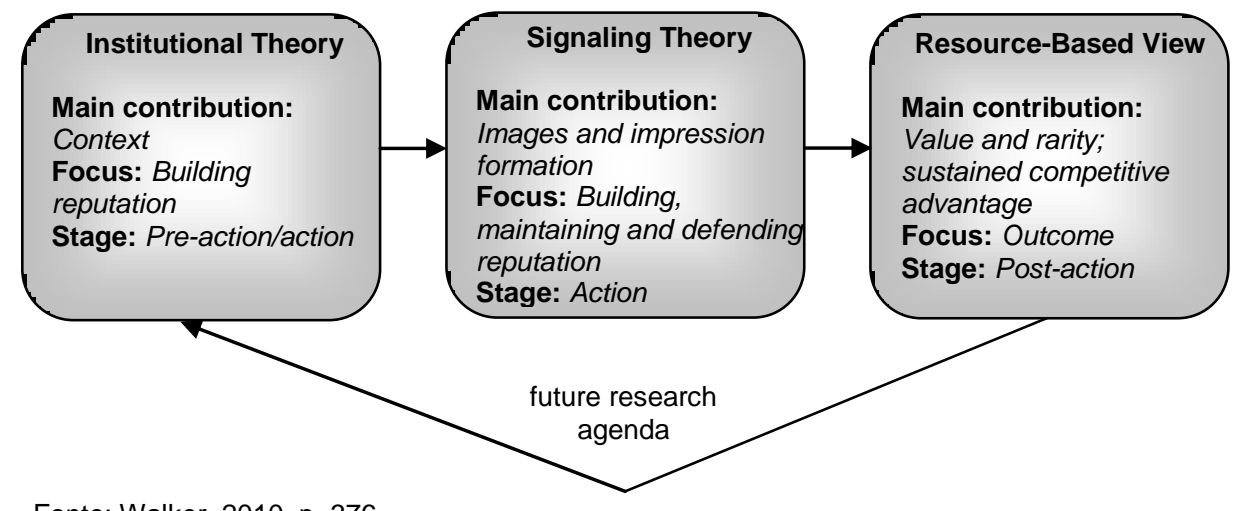

Fonte: Walker, 2010, p. 376

Per l'attivazione ed alimentazione costante di tale circolo virtuoso non può essere taciuto il contributo della Corporate Communication, dal momento che proprio attraverso il vettore comunicazionale l'impresa attiva contatti, gestisce relazioni, crea e sostiene la fiducia dei mercati (mediante messaggi e comportamenti) ed esercita condizionamenti sul contesto (tramite la loro decodifica).

All'interno di tale inquadramento concettuale e basandosi su un'analisi multidimensionale del costrutto corporate reputation, il presente lavoro propone una riflessione critica sulla "rilevanza strategica" della Total Corporate Communication in quanto "management function that offers a framework for the effective coordination of all internal and external communication with the overall purpose of establishing and maintaining favourable reputations with stakeholder groups upon which the organization is dependent" (Cornelissen, 2011, p. 5), al fine di definire un livello di reputazione ottimale da preservare e controllare per la creazione di valore d'impresa.

\section{Il contributo della comunicazione al processo di building reputation}

La reputazione di cui gode un'organizzazione si costituisce come fenomeno complesso nella sua analisi, alla cui rappresentazione contribuiscono numerose

4 In altre parole il capitale reputazionale si configura quale componente - non unica ma certamente rilevante per la creazione di ricchezza - del differenziale tra il valore di mercato dell'azienda ed i suoi asset tangibili. 
variabili, trovando anticipazione in una serie di esperienze pregresse degli attori del macro e del micro-ambiente ed affermazione nelle relazioni consolidate con i soggetti interessati dal rapporto, necessitando però di costanti riconferme (Winn et al., 2008). In altre parole, essa costituisce la sintesi di un vasto insieme di segnali circa il proprio agire strategico che l'organizzazione trasmette agli stakeholder nel corso del tempo, in modo sia esplicito che implicito, consentendogli di formulare previsioni sul comportamento futuro dell'impresa: la coerenza tra tali attese e gli effettivi comportamenti aziendali determina la formazione della corporate reputation (Nelli e Bensi, 2003).

L'instaurazione di un rapporto fiduciario concorre a migliorare la capacità dell'impresa di interazione con i propri stakeholder, sia all'interno del contesto aziendale - attenendo alla coesione e alla motivazione delle risorse umane, garantisce condizioni di equilibrio dinamico al sistema aziendale e favorisce l'ordinato svolgersi dei processi di gestione - che all'esterno - con riferimento alla credibilità e all'immagine dell'organizzazione (Pruzan, 2001; Furman, 2010; Otubanjo et al., 2010) presso l'ampia gamma di interlocutori attuali e potenziali, per mezzo di processi di comunicazione espressamente finalizzati a formare e sostenere la reputazione aziendale (Pastore e Vernuccio, 2008).

Evidentemente, il concetto si presta ad essere analizzato in una prospettiva multidimensionale, poiché al di là del sistema di identità visiva e dei messaggi veicolati dall'azienda (sistema di comunicazione aziendale), l'insieme dei suoi comportamenti e le performance del suo sistema di offerta presentano un'indubbia valenza comunicativa. Di conseguenza, la reputazione, che si configura quale "credibilità provata che consente di «dare un fondamento al diritto di chi promuove la comunicazione ad affermarla senza che alter (il ricevente) debba verificarla», costituisce l'alternativa, basata sulla fiducia, alla verifica diretta delle attività organizzative da parte del ricevente" (Gili, 2005, p. 25). Essa costituisce un vettore in grado di "incapsulare" la fiducia, diffondendola e difendendola (Boero et al., 2008; Ravasi e Gabbioneta, 2004).

Quale output di un processo di socializzazione, per la costruzione di opinioni condivise e di consenso sociale, la corporate reputation, basata su una fitta rete di scambio di informazioni, è in realtà il prodotto di un processo di attribuzione di senso all'agire di impresa, che avviene a livello sociale (Fombrum, 2001), ed è alimentato dalla condivisione e dalla socializzazione delle opinioni dei pubblici interessati.

Da questo punto di vista è agevole evidenziare un primo livello di influenza del vettore comunicazionale sul processo di reputation building, poiché se le attività di comunicazione non sono in grado di modificare la realtà aziendale, indubbiamente possono "orientare" l'impatto dei comportamenti dell'organizzazione (nel breve/medio periodo) sull'immaginario comune.

La reputazione è, infatti, un fenomeno collettivo, declinabile in relazione a diversi e ben identificabili gruppi di interesse, impegnati a riconoscere ed interpretare cosa l'impresa significa o rappresenta (Walsh et al., 2009; Walker, 2010). Essi, in relazione ad una esperienza diretta con l'impresa, o in conformità ai 
segnali informativi e valoriali ricevuti attraverso canali ufficiali e reti relazionali informali, procedono a riconoscere significatività ai messaggi diffusi, alla identità visiva e simbolica dell'organizzazione, alla sua cultura, alle immagini sostenute nel mercato.

Tale processo di sensemaking, di natura dinamica in quanto articolato nel corso del tempo, ha consistenza collettiva retrospettiva, poiché riferita ad avvenimenti già accaduti (Romenti, 2008). A completamento, è possibile attivare processi di sensemaking in prospettiva, ovvero rivolgendo l'attenzione a future opportunità di business, quale base decisionale per l'orientamento delle scelte strategiche (figura 2).

Fig. 2: Il processo di formazione della reputazione

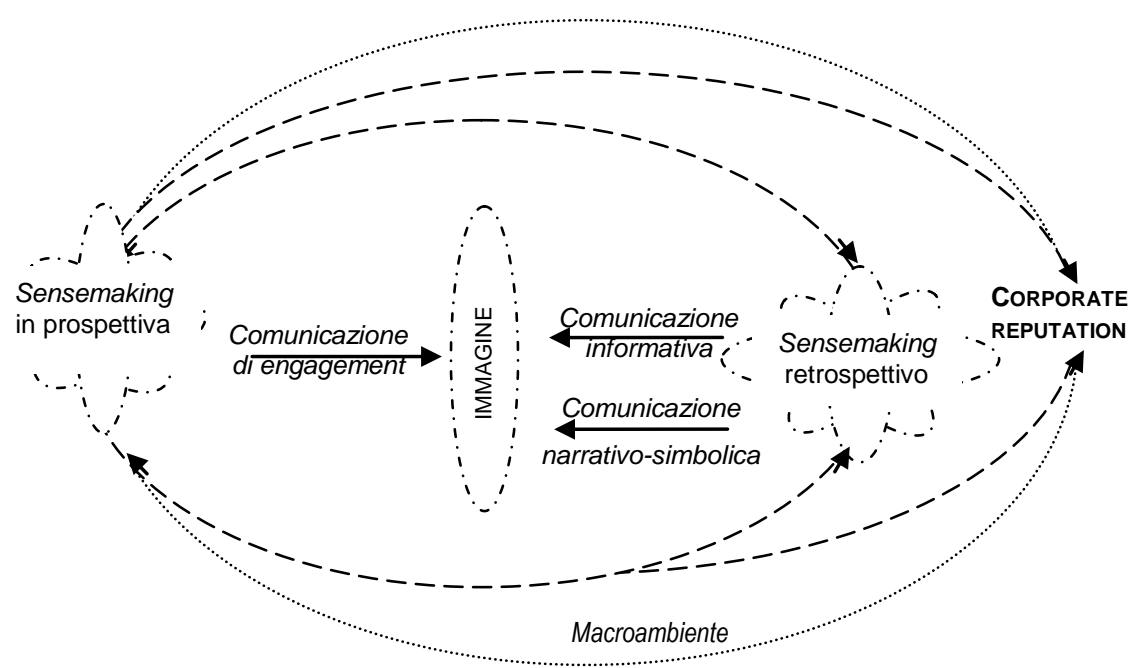

Fonte: ns. adattamento da Romenti, 2008, p. 13

Perché tale formulazione in prospettiva abbia compimento, è necessario che si realizzi un pieno coinvolgimento dei portatori di interessi, chiamati ad un confronto attivo con l'impresa sulle decisioni aziendali ${ }^{5}$.

In particolare, l'attività di comunicazione, intesa quale vettore di attivazione della collaborazione tra i soggetti interessati, diviene essenziale per la creazione dei setting organizzativi condivisi entro cui si generano tali anticipazioni reputazionali.

5 L'inclusione ed il coinvolgimento dei soggetti a vario titolo interessati, nella forma di consultazioni, cooperazioni, partnership ha il fine di sviluppare relazioni di profonda collaborazione e di condivisione di responsabilità con gli stakeholder sulla conduzione d'impresa. 
Nella formazione della reputazione aziendale, infatti, alla comunicazione viene riconosciuto un ruolo di divulgazione di informazioni di input per i processi decisionali e di diffusione dei risultati (comunicazione informativa), nonché di trasferimento all'esterno dei valori autentici dell'impresa (funzione narrativosimbolica). A tali aspetti si aggiunge un ruolo di alimentazione del processo di sensemaking in prospettiva, in termini di anticipazione di reputazione, a conferma che il costrutto è fortemente people-dependent, "viewed differently by different stakeholder group" (Ang e Wight, 2009, p. 21). Inoltre, non è da trascurare l'effetto prodotto da situazioni di disparità informativa, che portano alla sovraesposizione di alcune specifiche imprese, in grado di "occupare la scena" grazie ad una consistente dotazione di risorse, generando una maggiore notorietà nella mente del pubblico. Nella polarizzazione della dinamica comportamentale d'impresa tra saper far bene (being good in termini reputazionali) e farlo sapere (being known), il secondo termine sembra assumere, almeno oggi, un peso maggiore. Di conseguenza, la reputazione può essere definita anche in funzione della particolare dimensione di prominence, ovvero con riferimento al grado con cui un'organizzazione è collettivamente riconosciuta e si distingue all'interno del contesto competitivo (Rindova et al., 2005). Eventuali orientamenti strategici volti a conquistare/sostenere un elevato livello di prominence devono, tuttavia, essere continuamente valutati, in termini di costo/opportunità, commisurandoli al livello complessivo di reputazione di cui gode l'organizzazione. Non è detto, infatti, che una condizione di sovraesposizione comunicazionale produca sempre effetti positivi sul mercato; se una correlazione è evidenziabile quando le imprese possono vantare una reputazione robusta, utilizzando le competenze comunicative (in termini di prominence) quale strumento per una conferma della credibilità dell'organizzazione, già abbondantemente dimostrata e riconosciuta, diversamente può capitare in situazioni di sovraesposizione a carico di imprese in debito reputazionale. Queste ultime, infatti, rischiano di mettere in piazza performance, relazioni o percezioni dei diversi portatori di interesse poco lusinghiere, esponendo l'intera organizzazione ad un potenziale effetto valanga, generato ed alimentato proprio dalla sovraesposizione, laddove una strategia di basso profilo potrebbe consentire di operare concretamente per recuperare i gap sostanziali che ne affliggono l'operato.

In estrema sintesi, dunque, la gestione integrata di segni (comunicazione intenzionale ed esplicita), comportamenti e fatti osservabili e reti di relazioni - total corporate communication - consentono all'impresa di testimoniare il mantenimento delle promesse fatte agli stakeholder, sostenendone e rafforzandone la reputation (figura 3).

Invero, ogni processo di misurazione della reputazione si basa proprio su una valutazione dei comportamenti dell'impresa, così come sulla sua capacità di networking, attraverso lo studio dei rapporti instaurati con la comunità locale. In aggiunta, l'approccio multistakeholder proposto nel presente lavoro può completare la valutazione delle reti di relazioni. 
Fig. 3: Le categorie della corporate communication per costruire la reputazione

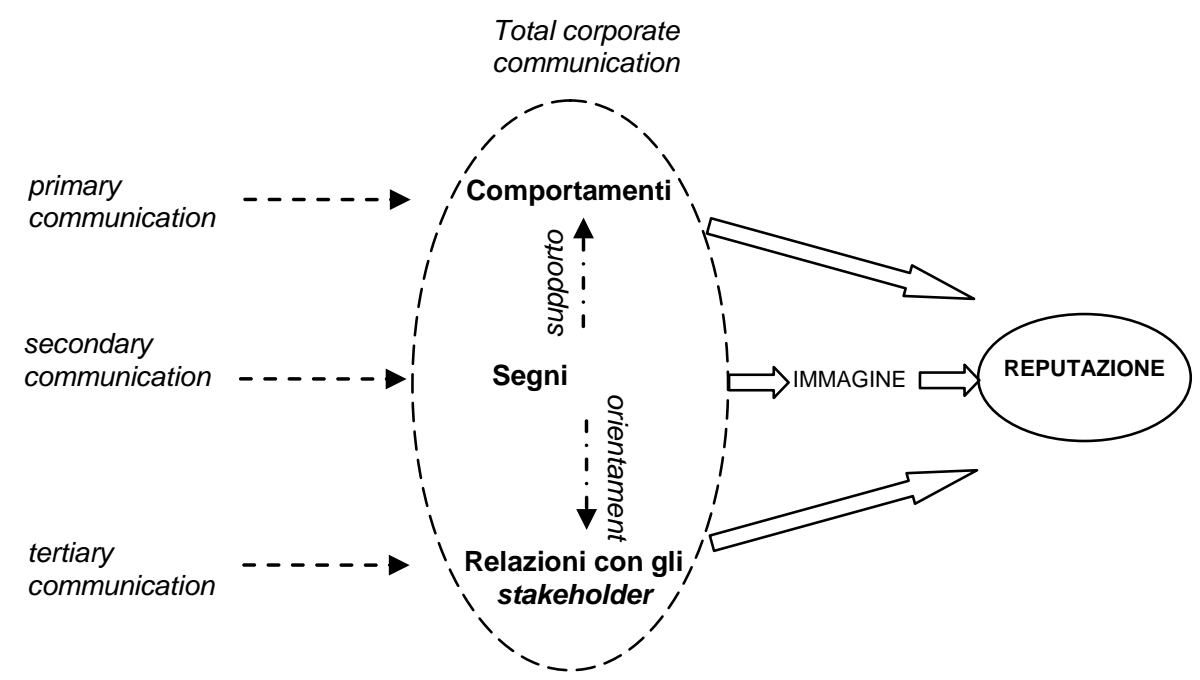

Fonte: ns. elaborazione da Mazzei e Gambetti, 2006, p. 10

In definitiva, dunque, la comunicazione esplicita ed intenzionale (i segni) può essere considerata - e valutata - quale elemento trasversale rispetto alle altre dimensioni componenti la reputazione d'impresa, fungendo da supporto per i comportamenti aziendali e con funzione di orientamento per le reti di relazioni: per un verso, infatti, essa rende trasparente, quindi percepibile, il valore offerto dall'impresa, a fronte dei costi sostenuti (riduzione dell'asimmetria informativa), i processi di generazione delle competenze ed i valori guida (Siano, 2001; Invernizzi e Romenti, 2005), creando un substrato per l'approvazione dei comportamenti d'impresa (primary communication).

Per altro aspetto, invece, la secondary communication ${ }^{6}$ esercita un potere di orientamento delle informazioni, conoscenze, opinioni, atteggiamenti relativi all'azienda da parte degli stakeholder e della collettività, contribuendo a consolidare la forza reputazionale dell'impresa quando il verso intrapreso dalla comunicazione implicita (tertiary communication) è convergente con quello della comunicazione intenzionale.

$6 \quad$ Al fine di incrementare positivamente la corporate reputation la comunicazione esplicita ed intenzionale deve presentare le seguenti caratteristiche: 1. grado di familiarità percepito; 2. consistenza nel tempo e nello spazio; 3. trasparenza; 4. distintività (memorabilità e posizionamento); 5. veridicità, contribuendo alla rappresentazione cognitiva di un metaconcetto che si costruisce e si gestisce nel lungo periodo (Mazzei e Gambetti, 2006). 


\section{Una verifica empirica: ipotesi e metodologia della ricerca}

All'interno del framework concettuale delineato, il presente lavoro si propone di esaminare il contributo della comunicazione intenzionale ed esplicita al costrutto corporate reputation, secondo un approccio multidimensionale, multistakeholder e time-based, dovendo necessariamente considerare come una quota di reputazione evidentemente si sottrae al controllo aziendale, legandosi alle percezioni, ovvero alle reazioni affettive ed emozionali dei soggetti interessati.

Partendo da un approccio alla corporate reputation quale percezione complessiva del modo di fare impresa da parte degli stakeholder, in termini di issues che influenzano la qualità delle relazioni di business (Gabbionata et al., 2007), è possibile individuare le principali componenti impegnate in un processo di reputation building, raggruppate in sei pilastri fondamentali, ovvero: emotional appeal; prodotti e servizi; performance reddituale e finanziaria; vision \& leadership; ambiente di lavoro; responsabilità sociale. Tali dimensioni (Fombrun et al., 2000), ed i relativi indicatori, costituiscono gli elementi fondamentali per la conduzione della misurazione della reputazione - Reputation Quotient (RQ) (Berens e Van Riel, 2004), cogliendo le percezioni dei differenti stakeholder, a valle della propria esperienza relazionale ${ }^{7}$.

Il capitale reputazionale di cui gode l'organizzazione imprenditoriale, determinato attraverso il calcolo del $\mathrm{RQ}^{8}$, fornisce al management e/o alla proprietà un utile strumento di monitoraggio del suo andamento nel tempo e, soprattutto, permette di esaltare i fattori strutturali e di comportamento strategico a più elevato impatto sul valore del RQ. Tutto ciò può essere meglio esplicitato ed analizzato applicando il modello delle aree di RQ di Cuomo-Metallo (figura 4).

Nel modello è possibile individuare un valore di demarcazione (Resistance Level per $\mathrm{RQ}=60^{9}$ ) al di sopra del quale l'impresa gode di una salda reputazione, ripartendosi in area granitica ed area stabile.

7 Nel presente lavoro è stato utilizzato un modello multistakeholder e multistadio, messo a punto dagli Autori per la misurazione del RQ: ogni livello si articola e si sviluppa secondo una visione incrementale sia della robustezza che dell'attendibilità della misura. Di conseguenza, si presentano di seguito i risultati ottenuti dalla versione ponderata (in relazione al peso di diversi stakeholder coinvolti nell'indagine) ed ampliata (considerando altri elementi determinanti) per la definizione della corporate reputation (Cuomo et al., 2012a).

8 L'espressione del RQ, riportato in centesimi, nasce da punteggi assegnati su scale metriche di facile ed oggettiva traduzione delle percezioni dei vari stakeholder, rendendo l'indicatore stesso confrontabile nel tempo e nello spazio e permettendone una valutazione generalizzabile.

9 In coerenza con il modello proposto da Harris Interactive, che esprime un giudizio generale sulla corporate reputation (score RQ $\geq 80$ : eccellente reputazione; 75-80: reputazione molto buona; 70-75: reputazione buona; 65-70: reputazione discreta), il modello proposto, a seguito di alcuni test di verifica effettuati, definisce il resistance level a $R Q=60$. 
RQ 80-100 Area granitica. È l'area in cui l'azienda vede assicurata una vera e propria riserva di reputazione. Ciò permette di sottolineare che l'investimento in reputazione, sia pure a produttività decrescente in quest'area, costruisce nel tempo delle vere e proprie barriere protettive verso l'area stabile, che fungono da ammortizzatori di eventuali spinte distruttive di valori reputazionali.

RQ 60-80 Area stabile. È caratterizzata da una reputazione forte e consolidata, che assicura all'impresa una significativa barriera protettiva verso eventi negativi e soprattutto una serie di vantaggi competitivi non solo verso i clienti (anche in termini di premium price) ma anche verso i finanziatori, disponibili ad erogare capitali sia di rischio che di finanziamento a tassi più contenuti; verso il personale generalmente più motivato e meno disponibile ad abbandonare l'azienda, la quale è in grado di attrarre risorse umane ad elevata professionalità; infine, verso i fornitori più aperti a rapporti cooperativi e collaborativi, soprattutto in termini di trasferimento del proprio know-how.

Fig. 4: Modello delle aree di RQ di Cuomo-Metallo

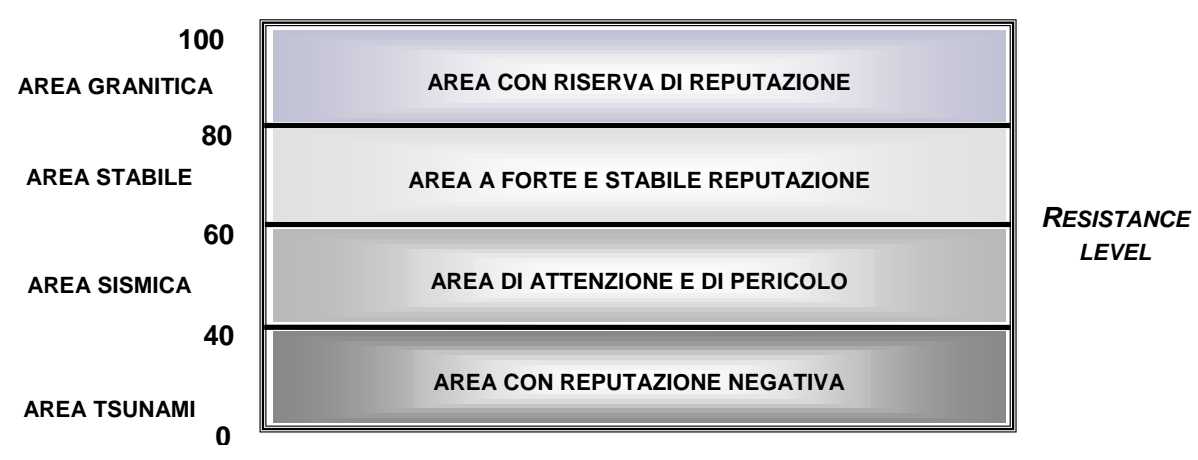

Fonte: Cuomo et al. (2012b)

Al di sotto del Resistance Level si posizionano le aree critiche reputazionali:

RQ 40-60 Area sismica. È l'area che contiene i valori di aziende in debito di reputazione e, quindi, in condizione di pericolo di sopravvivenza. Permanere a lungo in quest'area significa vivere quotidianamente il rischio di distruzione completa della reputazione con tutte le conseguenze strategiche ed operative, anche in termini di vitalità dell'impresa. Il potere contrattuale e competitivo di queste organizzazioni appare debole e scarsamente sostenibile, con gravi ripercussioni sulla redditività e sulla produzione di valore economico. Un management illuminato e/o una proprietà attenta dovranno porre in essere strategie di sviluppo del RQ per portare l'azienda nell'area stabile, o quantomeno evitare il baratro dello tsunami.

RQ 0-40 Area tsunami. L'azienda che entra in quest'area ha sostanzialmente distrutto il proprio patrimonio reputazionale e finisce per avere una reputazione negativa che coinvolge e condiziona le relazioni con i diversi stakeholder. Difficilmente troverà finanziatori disponibili ad apportare capitali e ad investire nei propri progetti, così come la clientela, che tenderà a spostare la propria domanda 
verso offerte alternative più affidabili; il personale sarà demotivato e pronto ad abbandonare l'azienda stessa ed i fornitori a rompere i rapporti di fornitura o a ribaltare sul prezzo e/o garanzie accessorie i maggiori rischi percepiti. Più semplicemente l'azienda con un RQ in quest'area è in balia di forze distruttive!

Pertanto, partendo da un'analisi di posizionamento delle organizzazioni in relazione al quoziente reputazionale, è possibile definire l'ipotesi di ricerca del presente lavoro, ovvero:

H.1: Esiste una relazione bidirezionale tra total corporate communication $e$ corporate reputation.

Da ciò, si articola una prima domanda, che definiamo di tipo retorico, dal momento che la letteratura scientifica ha risposto positivamente ${ }^{10}$ :

1.1 La comunicazione (intenzionale ed esplicita) contribuisce alla diffusione/variazione della reputazione d'impresa?

COMUNICAZIONE

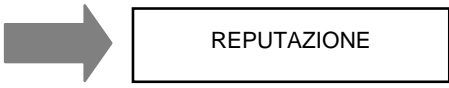

Infatti, molti studi sottolineano una relazione positiva tra la copertura mediatica offerta ad un'impresa e la sua reputazione (vedasi tra gli altri Carroll, 2011).

Tuttavia occorre precisare che, se per un verso la comunicazione contribuisce all'accrescimento degli intangible aziendali attraverso la divulgazione e la celebrazione del loro valore, essa stessa è alimentata, accresciuta, reinterpretata in relazione al valore di tali intangible, dal momento che l'audience si lascia a sua volta influenzare dal livello di reputazione della fonte della comunicazione per la decodifica dei messaggi, alterandone, seppur involontariamente, l'efficienza/efficacia.

Ciò porta alla formulazione della research question indagata:

1.2 A sua volta la corporate reputation influenza il processo di decodifica della comunicazione intenzionale ed esplicita da parte dei pubblici di riferimento? 1.3

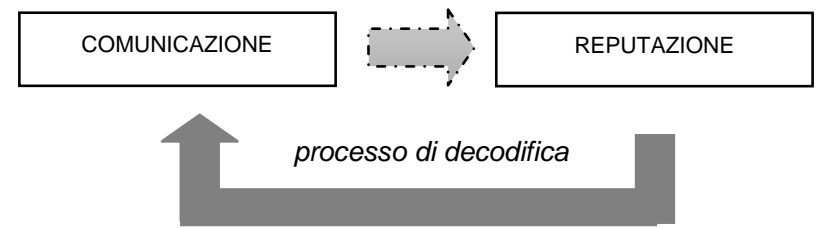

${ }^{10}$ La research question 1.1 viene riportata per articolare in maniera completa e puntuale l'ipotesi di ricerca, poichè è stata indicata una relazione bidirezionale tra total corporate communication e corporate reputation. 
Specie con riferimento alle organizzazioni imprenditoriali well known è interesse della ricerca indagare il fenomeno di resistenza verso messaggi che non risultano in linea con il personale (del singolo individuo) o collettivo (di tutti gli stakeholder) livello reputazionale attribuito all'impresa in questione. In tali situazioni è la reputazione di cui gode l'organizzazione presso i pubblici di riferimento a guidare il processo di decodifica del contenuto della comunicazione da parte dell'audience, trasformando un significato ostico in uno accettabile, in linea con le proprie credenze. Per un consumatore fedele, infatti, il costo cognitivo legato alla riattivazione del processo di sensemaking può risultare più elevato rispetto a quello impiegato per convincersi della possibilità di una interpretazione alternativa della comunicazione fruita. Per le organizzazioni ben sedimentate nell'immaginario collettivo, ciò è vero anche per l'intera comunità di riferimento.

Dal punto di vista delle imprese, mentre un disallineamento palese tra contenuto e/o attività di comunicazione e livello di reputazione attribuito alla fonte dagli stakeholder può essere governato per ripristinare il controllo della situazione, certamente maggiore attenzione deve essere posta sul processo di orientamento ed interpretazione dei contenuti della secondary communication, ad opera del capitale reputazionale d'impresa, in situazioni abituali ed ordinarie.

È possibile ipotizzare, infatti, che gli stakeholder, in special modo i consumatori - produttori attivi di senso e, quindi, co-creatori di valore - in funzione della credibilità accordata ad una impresa/brand, interpretino differentemente i segnali da essa emanati, utilizzando un maggior grado di indulgenza (per le imprese con reputazione in area stabile o granitica), o di inflessibile severità (nei confronti delle organizzazioni collocate in area sismica o tsunami).

Se è possibile osservare quanto descritto, non risulta più la comunicazione il vettore di influenza e sostegno della reputazione aziendale, ma, viceversa, è il posizionamento delle imprese in relazione al resistance level che direttamente incrementa o depaupera la comunicazione del suo intrinseco significato e valore.

Di conseguenza è necessario un ripensamento della collocazione strategica della comunicazione nell'area delle scelte di management.

Al fine di validare l'ipotesi, è stata realizzata una prima indagine sul campo, circoscritta ad alcune aziende alberghiere, senza pretesa di rappresentatività statistica del settore. Tale scelta è stata motivata sia dalla significatività del comparto per l'economia nazionale e locale (la Campania è settima per numero di strutture ricettive presenti sul territorio), sia perché la detenzione di un buon capitale reputazionale costituisce da sempre un asset distintivo per le strutture ricettive. La rilevanza delle attività comunicazionali per la reputazione dell'offerta ricettiva costituisce un aspetto di criticità per il sistema turistico-alberghiero.

La ricerca esplorativa (Barile e Metallo, 2002; Molteni e Troilo, 2003), si è basata su un panel costituito da 6 alberghi collocati in provincia di Salerno e confrontabili per categoria di appartenenza (4/5 stelle), al fine di poter ipotizzare una comunanza di elementi valutativi di base:

- capacità ricettiva,

- professionalità degli operatori, 
- qualificazione del management anche in presenza di imprese familiari,

- accoglienza e gestione dell'ospite,

- cura dell'atmosfera (arredamento, pulizia,ecc.),

- varietà dei servizi offerti.

Output dell'analisi, rispondendo è l'esplicitazione del legame esistente tra la struttura dell'organizzazione imprenditoriale, la comunicazione effettuata e la sua reputazione, evidenziando eventuali nessi di causa-effetto. Il risultato atteso è l'identificazione dei collegamenti rilevanti per la descrizione della soglia critica reputazionale. La fase di rilevazione è schematizzata in figura 5 .

Fig. 5: Le fasi operative della ricerca

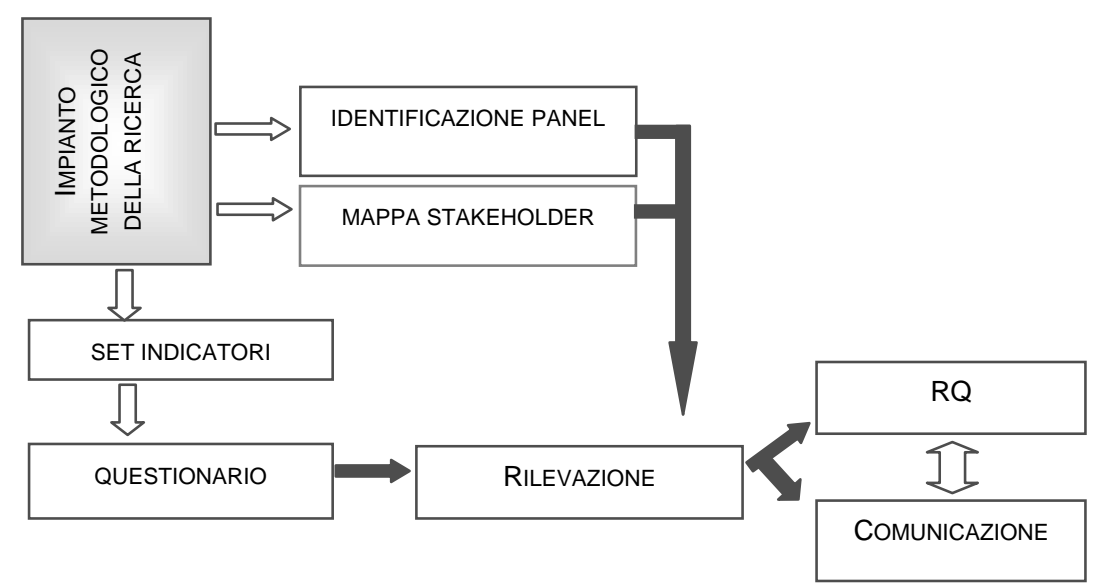

Fonte: ns. elaborazione

Per la rilevazione dei dati sul campo è stato predisposto un questionario, volto a cogliere per ciascuna struttura ricettiva l'influenza delle attività di comunicazione sulla scelta della struttura stessa, da commentare alla luce del RQ misurato con il citato approccio multistakeholder (Cuomo et al., 2012b) ${ }^{11}$. Si precisa, quindi, che in

11 Per rilevare il RQ per ciascuna struttura alberghiera sono stati somministrati 30 questionari, customizzati per stakeholder, intervistando: esponenti in rappresentanza del sistema proprietario; dipendenti per il riferimento agli stakeholder interni; fornitori; clienti per cogliere le percezioni degli stakeholder esterni. Per la valutazione della attività di comunicazione sono stati somministrati ulteriori 30 questionari per ciascun albergo, intervistandone i soli ospiti presenti. Le rilevazioni sono state condotte a luglio 2011. Dipendenti e fornitori sono stati selezionati estraendo i nominativi da intervistare in maniera casuale all'interno di una lista fornita dalla struttura alberghiera. Analogamente, $i$ clienti sono stati selezionati casualmente tra gli ospiti presenti nelle strutture ricettive nel periodo in cui è stata condotta l'indagine. I dati sono stati rilevati tramite interviste personali e registrati dall'intervistatore sull'apposita scheda di rilevazione. 
questo lavoro si incrociano i risultati per la rilevazione del RQ con quelli relativi al monitoraggio delle attività di comunicazione.

\section{I risultati della ricerca}

Al fine di consentire una lettura esaustiva delle informazioni rilevate on field è opportuno presentare sinteticamente i risultati ottenuti in ordine al RQ di cui godono le imprese censite, desunto attraverso un modello multistadio, che procede per livelli progressivi di approfondimento della reputazione di un'organizzazione ${ }^{12}$ (tabella 1).

Tab. 1: Il capitale reputazionale delle strutture alberghiere (in percentuale)

\begin{tabular}{|c|c|c|c|}
\hline $\begin{array}{c}\text { Struttura } \\
\text { alberghiera } \\
\text { (codice) }\end{array}$ & $\begin{array}{c}\text { Primo livello } \\
\text { di RQ }\end{array}$ & $\begin{array}{c}\text { Secondo } \\
\text { livello di RQ }\end{array}$ & $\begin{array}{c}\text { Terzo livello di } \\
\mathbf{R Q}\end{array}$ \\
\hline CAP & 79,46 & 72,10 & 57,76 \\
\hline GRH & 70,57 & 66,78 & 53,97 \\
\hline CER & 82,49 & 84,99 & 67,39 \\
\hline HRA & 72,84 & 71,68 & 60,59 \\
\hline LBH & 76 & 71,39 & 60,07 \\
\hline PIN & 76,15 & 74,13 & 66,07 \\
\hline
\end{tabular}

Fonte: ns. elaborazione

I risultati esposti devono essere messi a confronto con il posizionamento di ciascuna struttura rispetto al relativo ambiente concorrenziale, evidenziando un miglioramento da parte degli alberghi che, sulla base del terzo livello di RQ, sono stati collocati in zona di pericolo (CAP in particolare fa registrare un notevole apprezzamento in termini di posizionamento; anche GRH vede migliorare la propria collocazione figura 6). Solo CER, struttura che presentava il punteggio più elevato per il terzo livello di RQ, non conferma il suo posizionamento nella percezione degli stakeholder intervistati. Si osservi, tuttavia, come tali cambiamenti potrebbero essere

12 Nel modello di misurazione del RQ proposto il primo livello di $R Q$ prende a riferimento gli item suggeriti dal Reputation Institute arricchendoli di un ulteriore item per la misurazione delle performance finanziarie; il secondo livello di $R Q$, invece, ingloba il meccanismo della ponderazione dei diversi pilastri in relazione ali interlocutori aziendali coinvolti nell'indagine (multistakeholder approach); il terzo livello di $R Q$, in relazione all'importanza dei vari pilastri per i diversi portatori di interesse, prende in considerazione anche altre indicazioni e variabili influenti sulla percezione del valore reputazionale (p.e., longevità dell'insegna; struttura proprietaria, ecc.); infine, si segnala un quarto livello di $R Q_{2}$ ottenuto tramite la fusione del terzo livello del RQ e la web reputation (WR) delle organizzazioni, misurata con il supporto di tool che consentono di eseguire dei monitoraggi anche in tempo reale. Per approfondimenti sul modello multistadio accennato vedasi ampiamente: Cuomo et al., 2012b. 
collegati alle differenze dell'arena competitiva in cui le imprese alberghiere operano.

Fig. 6: Posizionamento degli alberghi secondo la percezione degli stakeholder

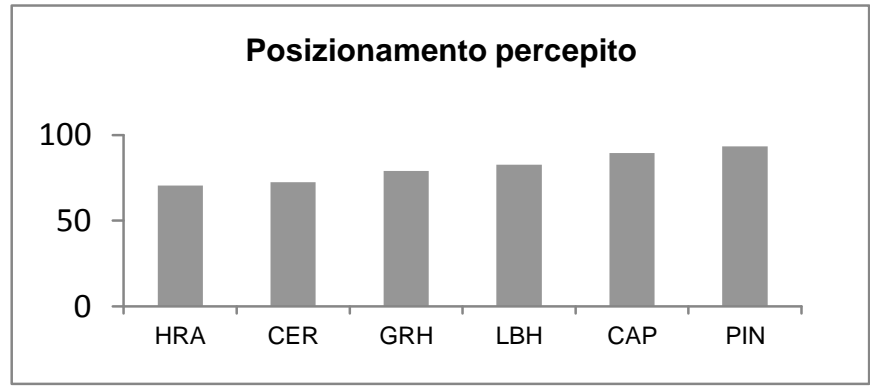

Fonte: ns. elaborazione

Infatti, sia HRA che CER sono collocate in aree caratterizzate da un'elevata concentrazione di alberghi di eccellenza (rispettivamente la Costiera Amalfitana e quella Cilentana), rendendo più complessa l'applicazione di una strategia di differenziazione.

In generale, i risultati ottenuti confermano l'influenza dell'ambiente esterno sulle percezioni degli stakeholder. Di conseguenza, si può assumere che un elevato livello di reputazione non deve essere considerato come una variabile stand-alone (autonoma), bensì relativa, influenzata (anche in termini percettivi) dall'intensità e modalità competitiva di settore.

Infine, con l'intento di rendere l'analisi ancora più attendibile e robusta, è stato individuato un quarto livello di RQ, che prende in considerazione anche la dimensione della reputazione online. Oggigiorno la potente accelerazione della diffusione di Internet ha raggiunto una tale massa critica da imporre il web come uno dei media di riferimento per la formazione di opinioni e la valutazione dei comportamenti, oltre che dei prodotti/servizi, delle organizzazioni. Pertanto, il raffronto con la percezione online dell'azienda da parte degli utenti web diviene attualmente una tematica essenziale con cui confrontarsi, considerando la reputazione dell'organizzazione emergente online, non solo attraverso i siti istituzionali dell'impresa/brand, ma anche per mezzo di blog, chat, forum tematici, wiki e social network, pronti ad offrire all'opinione pubblica una diversa versione della realtà aziendale ${ }^{13}$.

13 Genericamente ed a seconda della finalità di indagine, per l'analisi della web reputation si può effettuare un monitoraggio in termini di brand presence, p.e. conteggiando il numero di citazioni e post su una marca, con analisi delle fonti (dove se ne parla e chi ne parla), se ne analizzano i topic, ovvero i concetti ricorrenti, le tematiche più discusse, le 
Nello studio presentato, prendendo in considerazione i commenti sugli alberghi esaminati rilasciati dai turisti sul sito web www.tripadvisor.it ${ }^{14}$ (considerando soltanto le opinioni espresse dai clienti) è stato calcolato il punteggio raggiunto da ogni struttura, pesandolo e comparandolo con i punteggi del terzo livello di RQ, prendendo in considerazione un solo tipo di portatore di interessi (i clienti) allo scopo di rendere il confronto quanto più affidabile possibile (GRH: 52,59; HRA: 57,96; LBH: 59,78; PIN: 63,82; CAP: 60,85; CER: 61,79). Sulla base dei giudizi dei clienti, i risultati ottenuti dalle valutazioni presentate in Trip Advisor, ancorché leggermente più contenute (GRH: 48,69; HRA: 51,70; LBH: 56,02; PIN: 56,09; CAP: 51,90; CER: 59,50) possono essere considerate abbastanza in linea con il terzo livello di RQ (solo clienti). Pertanto, dalla fusione dei due indicatori precedenti (punteggio desunto da Trip Advisor e terzo livello di RQ opportunamente ponderati) si è misurato il RQ di quarto livello. Al fine di potere confrontare questo risultato con i punteggi ottenuti in relazione agli altri indicatori, è utile fare riferimento alla tabella 2 .

Tab. 2: Confronto tra indicatori

\begin{tabular}{|c|c|c|c|c|}
\hline $\begin{array}{c}\text { Struttura } \\
\text { alberghiera } \\
\text { (codice) }\end{array}$ & $\begin{array}{c}\text { Primo livello } \\
\text { di RQ }\end{array}$ & $\begin{array}{c}\text { Secondo } \\
\text { livello di RQ }\end{array}$ & $\begin{array}{c}\text { Terzo } \\
\text { livello di } \\
\mathbf{R Q}\end{array}$ & $\begin{array}{c}\text { Quarto } \\
\text { livello di } \\
\mathbf{R Q}\end{array}$ \\
\hline CAP & 79,46 & 72,10 & 57,76 & 65,86 \\
\hline GRH & 70,57 & 66,78 & 53,97 & 56 \\
\hline CER & 82,49 & 84,99 & 67,39 & 69,52 \\
\hline HRA & 72,84 & 71,68 & 60,59 & 62,73 \\
\hline LBH & 76 & 71,39 & 60,07 & 66,17 \\
\hline PIN & 76,15 & 74,13 & 66,07 & 70,47 \\
\hline
\end{tabular}

Fonte: ns. elaborazione

Infine, per valutare l'efficacia della comunicazione intenzionale ed esplicita ne è stato indagato il livello di esposizione da parte dei clienti; pertanto, dopo aver proceduto ad effettuare una mappatura degli strumenti di comunicazione di marketing utilizzati da ciascuna struttura ${ }^{15}$, è stato sollecitato il ricordo spontaneo degli ospiti. Ciò che rileva ai fini dell'indagine, infatti, non è la quantità/qualità delle attività di comunicazione pianificata ed implementata dall’impresa (comportamento

conversazioni più dibattute ed il sentiment, cioè in che modo se ne parla. Confrontando $i$ volumi di buzz ed il sentiment con riferimento alla singola organizzazione, si può costruire una mappa del rischio reputazionale online. Vi sono, inoltre, siti che raccolgono, in base ad una serie di indicatori stabiliti come rilevanti, le percezioni degli stakeholder circa il comportamento delle imprese.

14 TripAdvisor è un sito web dedicato ai viaggi, che in soli due anni ha raddoppiato le recensioni pubblicate dagli utenti, passando da 25 milioni nel 2009 ai 50 milioni del luglio 2011.

15 Tale mappatura è avvenuta sia attraverso interviste dirette alla proprietà/management, sia attraverso l'analisi dei rispettivi siti internet. 
comunicazionale dell'emittente), quanto l'effettiva capacità di raggiungere i propri interlocutori (visibilità presso i pubblici) ed orientarne le percezioni (capacità di persuasione). Ebbene, eccezion fatta per la consultazione del sito $w e b^{16}$, sorprenderà verificare che, pur in presenza di una clientela nazionale ed estera, la diffusione dell'offerta alberghiera è stata "affidata" al passaparola, strumento di comunicazione - non intenzionale - efficiente in termini di costo/contatto, ma difficilmente controllabile dall'impresa (tabella 3 e 4$)^{17}$.

Tab. 3: Attività e strumenti di comunicazione off line (solo $1^{a}$ opzione)

\begin{tabular}{|c|c|c|c|c|c|c|c|c|c|}
\hline \multirow{2}{*}{$\begin{array}{c}\text { Struttura } \\
\text { alberghiera } \\
\text { (codice) }\end{array}$} & Spot & Cartellonistica & Brochure & $\begin{array}{c}\text { Attività } \\
\text { promozionali }\end{array}$ & Sponsorizzaz. & $\begin{array}{c}\text { Passa } \\
\text { parola }\end{array}$ & $\begin{array}{c}\text { Com. } \\
\text { Tour } \\
\text { operator }\end{array}$ & Catalogo & Inserto \\
\hline GRH & $\mathbf{0}$ & $\mathbf{0}$ & $\mathbf{2}$ & $\mathbf{0}$ & $\mathbf{2}$ & $\mathbf{6}$ & $\mathbf{1}$ & $\mathbf{1}$ & $\mathbf{0}$ \\
\hline CAP & $\mathbf{2}$ & $\mathbf{2}$ & $\mathbf{2}$ & $\mathbf{4}$ & $\mathbf{2}$ & $\mathbf{9}$ & $\mathbf{0}$ & $\mathbf{2}$ & $\mathbf{1}$ \\
\hline HRA & $\mathbf{0}$ & $\mathbf{1}$ & $\mathbf{2}$ & $\mathbf{1}$ & $\mathbf{0}$ & $\mathbf{4}$ & $\mathbf{6}$ & $\mathbf{2}$ & $\mathbf{0}$ \\
\hline LBH & $\mathbf{0}$ & $\mathbf{5}$ & $\mathbf{2}$ & $\mathbf{1}$ & $\mathbf{0}$ & $\mathbf{5}$ & $\mathbf{3}$ & $\mathbf{1}$ & $\mathbf{1}$ \\
\hline PIN & $\mathbf{0}$ & $\mathbf{1}$ & $\mathbf{1}$ & $\mathbf{4}$ & $\mathbf{0}$ & $\mathbf{5}$ & $\mathbf{0}$ & $\mathbf{0}$ & $\mathbf{0}$ \\
\hline CER & $\mathbf{0}$ & $\mathbf{6}$ & $\mathbf{5}$ & $\mathbf{4}$ & $\mathbf{0}$ & $\mathbf{6}$ & $\mathbf{4}$ & $\mathbf{3}$ & $\mathbf{1}$ \\
\hline
\end{tabular}

Fonte: ns. elaborazione

Tab. 4: Attività e strumenti di comunicazione online

\begin{tabular}{|c|c|c|c|c|}
\hline \multirow{2}{*}{$\begin{array}{c}\text { Struttura } \\
\text { alberghiera } \\
\text { (codice) }\end{array}$} & \multicolumn{4}{|c|}{ Comunicazione online } \\
\cline { 2 - 5 } & $\begin{array}{c}\text { Sito } \\
\text { web }\end{array}$ & Banner & Pubblicità & Email \\
\hline GRH & $\mathbf{1 0}$ & $\mathbf{3}$ & $\mathbf{0}$ & $\mathbf{0}$ \\
\hline CAP & 9 & 3 & 2 & 1 \\
\hline HRA & $\mathbf{7}$ & $\mathbf{0}$ & $\mathbf{1}$ & $\mathbf{0}$ \\
\hline LBH & $\mathbf{8}$ & $\mathbf{1}$ & $\mathbf{2}$ & $\mathbf{1}$ \\
\hline PIN & $\mathbf{9}$ & $\mathbf{0}$ & $\mathbf{0}$ & $\mathbf{1}$ \\
\hline CER & $\mathbf{7}$ & $\mathbf{0}$ & $\mathbf{1}$ & $\mathbf{2}$ \\
\hline
\end{tabular}

Fonte: ns. elaborazione

Ancora nel terzo millennio la rete più o meno estesa e difficilmente verificabile dei contatti personali rimane uno dei più potenti ed efficaci mezzi di divulgazione delle informazioni, seppur spesso parziali e viziate dalla esperienza soggettiva. Naturalmente, tale processo virale in cui i consumatori si trasformano in attori della comunicazione trova nel web la sua piattaforma di divulgazione ottimale, permettendo in poco tempo il raggiungimento di un valore critico di diffusione,

16 Tale attività è svolta dalla gran parte degli intervistati, sia per raccogliere informazioni aggiuntive sulla struttura che per fruire del servizio di prenotazione.

17 I dati riportati nelle tabelle 3 e 4 sintetizzano i risultati ottenuti attraverso le interviste dirette ad un gruppo di 30 turisti per struttura alberghiera (solo $1^{\mathrm{a}}$ opzione) e anche quelli derivanti dall'analisi dei rispettivi siti web. 
superato il quale la comunicazione tende ad autoalimentarsi in un processo di crescita esponenziale.

Ciò nondimeno, anche se difficilmente gestibile dall'impresa il virus del passaparola può essere innescato o aiutato in una prima fase di diffusione anche dall'emittente, attraverso un consapevole, seppur discreto e non invasivo, utilizzo degli altri strumenti di comunicazione di marketing. Tale strategia, tuttavia, non sembra essere adeguatamente sfruttata dal panel di alberghi coinvolti nell'indagine, dal momento che, ad eccezione del sito web, esiguo e marginale sembra essere il ricorso alle altre forme di comunicazione online (banner, email marketing, pubblicità/promozioni sul web), con contenuto appeal sui potenziali fruitori. In generale, il dialogo con la propria audience è affidato agli strumenti tradizionali della comunicazione off line, prediligendo cataloghi vacanze e tour operator o attivando forme e strumenti con valenza territoriale (cartellonistica e brochure). È da notare, però, almeno affidandosi alle percezioni dei clienti intervistati, che la pressione comunicativa messa in campo dalle strutture interessate non risulta essere particolarmente intensa (figura 7).

Fig. 7: Intensità della comunicazione (attività/strumenti ricordati dai clienti)

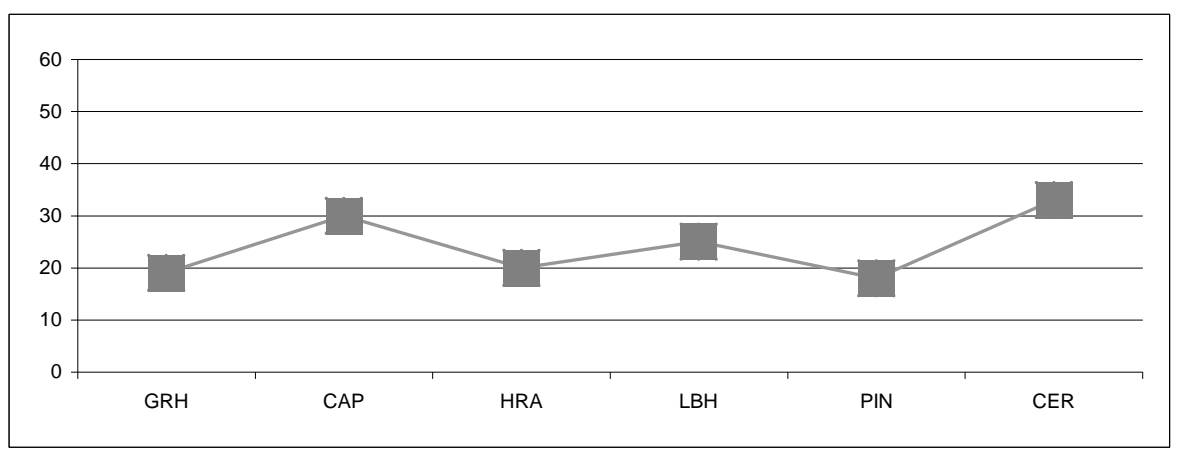

Fonte: ns. elaborazione

Benché modesta, comunque, la comunicazione risulta essere affidabile, credibile ed adeguata, facendo registrare una pressoché totale soddisfazione da parte degli ospiti, grazie ad un completo allineamento dei contenuti informativi/persuasivi con l'esperienza fruita.

\section{Prospettive future ed implicazioni di management}

Prendendo spunto dalle evidenze empiriche illustrate, è possibile sviluppare alcune considerazioni conclusive di ordine critico ed applicativo soprattutto di tipo strategico, pur tenendo ben a mente i limiti dell'analisi empirica proposta, derivanti 
da una descrizione statica e non dinamica del fenomeno indagato ed alla selezione degli stakeholder interessati dall'indagine, che in fase finale (per indagare gli strumenti di comunicazione) sono stati identificati nei soli clienti.

Ritornando, invece, ai risultati dell'indagine, per quanto riguarda il posizionamento delle aziende ricettive relativamente al RQ di quarto livello si ha la seguente rappresentazione (figura 8).

Fig. 8: Posizionamento degli Hotel censiti in base al RQ misurato

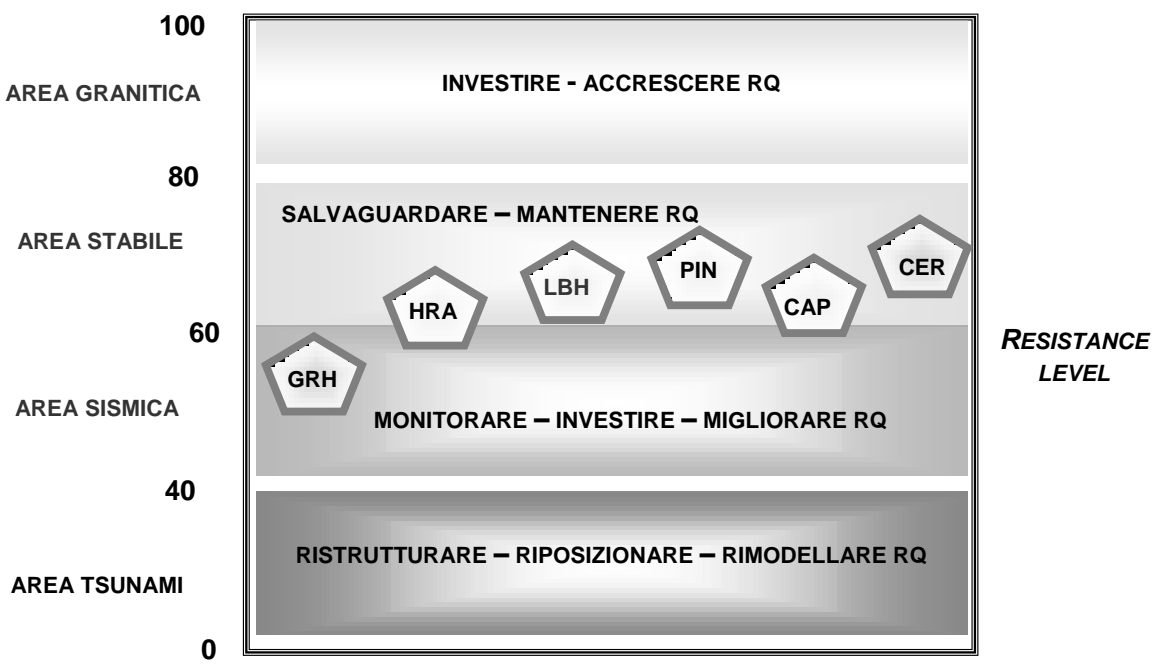

Fonte: ns. elaborazione

Il panel oggetto di osservazione è composto da strutture ricettive che vantano in alcuni casi un discreto capitale reputazionale (PIN, CER, LBH); ricordando che la corporate reputation si sedimenta nel tempo considerando i comportamenti dell'organizzazione, le reti di relazioni con gli stakeholder e la comunicazione intenzionale ed esplicita, è interessante effettuare delle ulteriori considerazioni mettendo in relazione quest'ultima (sostanziata nell'impegno in comunicazione delle strutture $^{18}$ ) con il RQ.

Dal momento che le strutture indagate, ad eccezione di GRH ${ }^{19}$, presentano un RQ medio, pur in presenza di un impegno in comunicazione contenuto, è possibile

18 L'indicatore è stato creato considerando gli strumenti fruiti dai clienti in relazione a tutti gli strumenti a disposizione delle strutture ricettive, ottenendo: CRH 16\%; CAP 25\%; HRA $17 \%$; LBH $21 \%$; PIN 4\%; CER $28 \%$.

19 In questo caso è necessario escludere qualsiasi tipo di informazione sulla reputazione dell'impresa nell'attività di comunicazione, che comunque non deve esporre eccessivamente la struttura finché non ne sia migliorato il posizionamento in termini reputazionali. 
affermare che, almeno nei casi osservati, non è l'attività di comunicazione esplicita ed intenzionale ad influenzare il RQ (figura 9); piuttosto, è la comunicazione attivata dalle strutture che viene ad essere decodificata in funzione dei comportamenti dell'impresa e delle reti di relazioni attivate, sostenute in primis dal buzz messo in azione dagli altri ospiti.

Fig. 9: Impegno riconosciuto in comunicazione e $R Q$

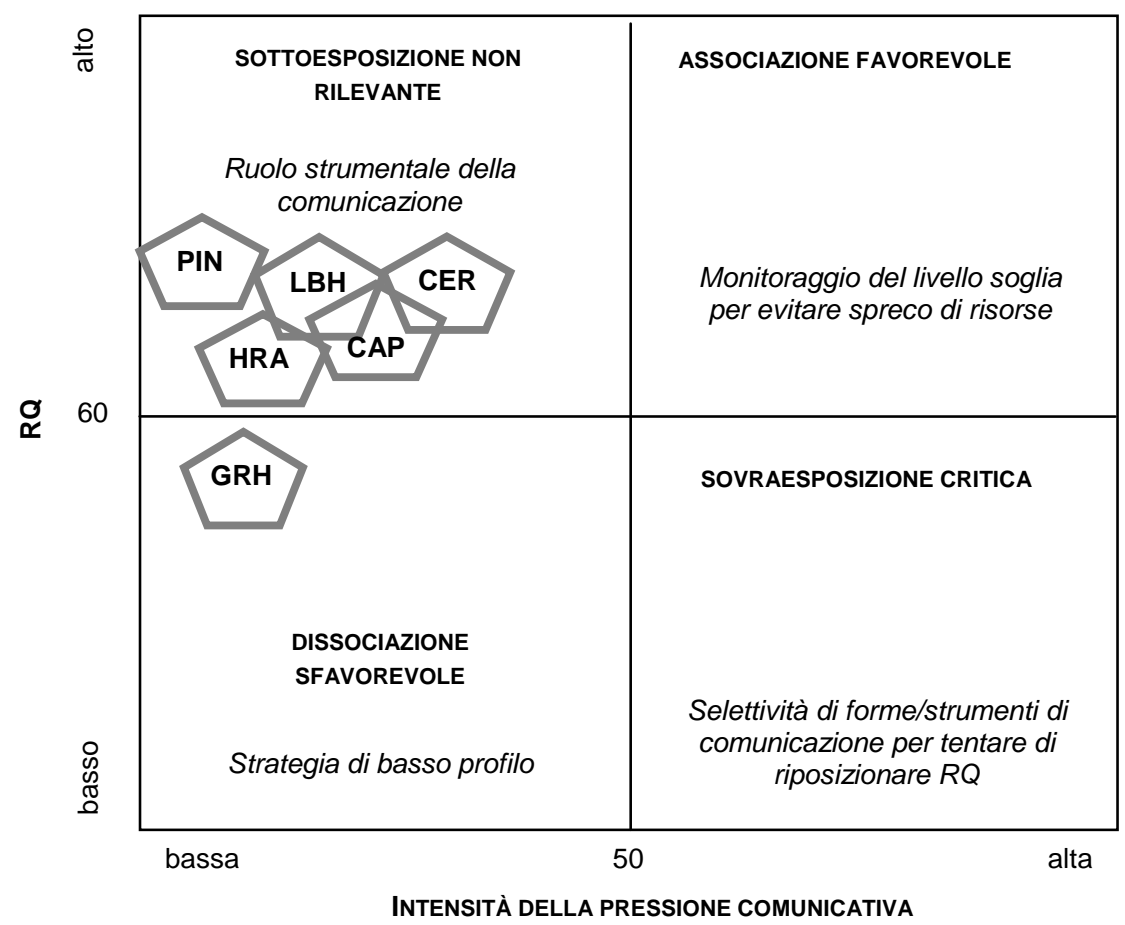

Fonte: ns. elaborazione

La reputazione di cui godono le strutture ricettive crea un contesto interpretativo favorevole che gli strumenti di comunicazione riescono solo successivamente a capitalizzare. L'assenza di una vera e propria strategia di comunicazione nelle strutture indagate, tuttavia, suggerisce più in generale la necessità di un ripensamento critico del ruolo assegnato alle attività di comunicazione all'interno del processo strategico d'impresa.

Nel dialettico confronto tra i poli being good e being known entro cui le organizzazioni devono scegliere il proprio posizionamento in termini reputazionali, evidentemente la propensione deve essere sbilanciata verso il primo termine del continuum. Anzi, laddove le imprese si trovassero in aree critiche, con dispersione di reputazione, una eccessiva collocazione sotto i riflettori del mercato, ottenuta con strategie di comunicazione aggressive o impegnative in termini finanziari, potrebbe 
portare alla citata e non sempre positiva sovraesposizione critica. Invece, laddove l'impresa possa contare su un livello reputazionale soddisfacente, deve dosare sapientemente gli investimenti in comunicazione, selezionando le forme ed i mezzi idonei ad interloquire con i differenti stakeholder, in modo da amplificare, finché possibile, il buon operato dell'organizzazione, pur nella consapevolezza che, raggiunto un livello soglia di ottimale presenza (quantitativa e qualitativa) presso i propri interlocutori, la strategia di dialogo con gli stakeholder non può che essere di mantenimento, rischiando in caso diverso di sprecare risorse ed indurre un effetto di assuefazione e distorsione dell'attenzione da parte dei pubblici (figura 10).

Fig. 10: Relazione tra intensità della pressione comunicativa e livello di $R Q$

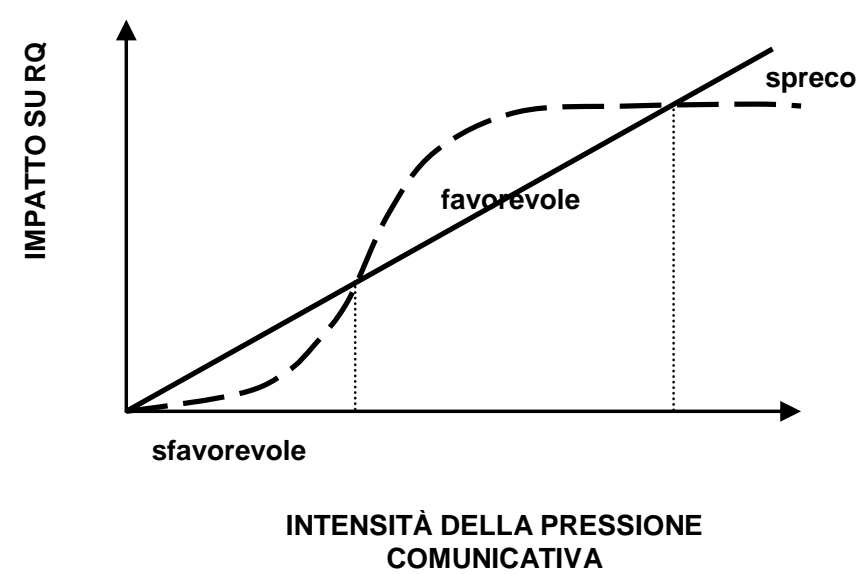

Fonte: ns. elaborazione

In altre parole, è possibile affermare che: "la comunicazione sostiene ciò che è sostenibile e non sostiene ciò che non è sostenibile" e riesce a costituire un plus per l'ottenimento di vantaggi competitivi se, e solo se, può contare su performance tali da assicurare la creazione di valore.

Le riflessioni proposte, comunque, senza alcuna pretesa di avere carattere di completezza, né di esaustività, suggeriscono una diversa ottica per la valutazione e l'utilizzo, specie in momenti di crisi, della comunicazione nelle scelte di management legate alla sopravvivenza ed alla continuità delle imprese, dal momento che è la reputazione delle organizzazioni imprenditoriali (e non viceversa) che qualifica l'interdipendenza ed i flussi comunicativi tra le componenti sia interne che esterne al sistema, suggerendo, non da ultimo, possibili evidenze sulla variazione del valore economico aziendale. 


\section{Bibliografia}

ANG S.H., WIGHT A.M. (2009), "Building Intangible Resources: The Stickiness of Reputation”, Corporate Reputation Review, vol. 12, n. 1, pp. 21-32.

BACCARANI C. (2008), “What does ethical behaviour mean in management activities?", The TQM Journal, vol. 20, n. 2, pp. 154-165.

BARILE S., METALLO G. (2002), Le ricerche di mercato, Giappichelli, Torino.

BARNEY J. (1991), "Firm resources and sustained competitive advantage", Journal of Management Studies, vol. 17, n. 1, pp. 99-120.

BERENS G., VAN RIEL C. (2004), "Corporate Associations in the Academic Literature: Three Main Streams of Thought in the Reputation Measurement Literature", Corporate Reputation Review, vol. 7, n. 2, pp. 161-178.

BOERO R., BRAVO G., CASTELLANI M., LAGANÀ F., SQUAZZONI F. (2008), "La reputazione come vettore di fiducia e cooperazione nei sistemi socio-economici: alcune evidenze sperimentali”, Dipartimento di Studi Sociali PAPERS, Brescia, n. 5, pp. 1-25.

CARROLL C. E. (edited by) (2011), Corporate Reputation and the News Media: AgendaSetting Within Business News Coverage in Developed, Emerging, and Frontier Markets, Routledge, New York.

CORNELISSEN J. (2011), Corporate Communication. A Guide to Theory and Practice, Sage Pubblications Ltd (third edition), London.

CUOMO M.T., TORTORA D., METALLO G. (2012a), “A multistakeholder approach for measuring corporate reputation. A case study survey of italian local smes", Conference Proceedings of 2012 Global Marketing Conference, July 19-22, Coex, Seoul, Republic of Korea.

CUOMO M.T., TORTORA D., METALLO G. (2012b), "La misurazione multilivello e multistakeholder della corporate reputation", Rassegna economica, n. 1, pp. 165-181.

FOMBRUN C., SHANLEY M. (1990), "What's in a name? Reputation building and corporate strategy", Academy of Management Journal, vol. 33, n. 2, pp. 233-258.

FOMBRUN C.J. (2001), "Corporate reputations as economic assets", in Hitt M.A., Freeman R.E., Harrison J.S. (edt.), The Blackwell Handbook of Strategic Management, UK: Blackwell Publishers, Oxford.

FOMBRUN C.J., GARDBERG N., SEVER J. (2000), "The Reputation Quotient: A multistakeholder measure of corporate reputation", Journal of Brand Management, vol. 7, n. 4, pp. 241- 255.

FOMBRUN C.J., VAN RIEL C. (1997), "The Reputational Landscape", Corporate Reputation Review, vol. 1, n. 1, pp. 5-13.

FURMAN D.M. (2010), "The Development of Corporate Image: A Historiographic Approach to a Marketing Concept", Corporate Reputation Review, vol. 13, n. 1, pp. 63-75.

GABBIONETA C., RAVASI D., MAZZOLA P. (2007), "Exploring the Drivers of Corporate Reputation: A Study of Italian Securities Analysts", Corporate Reputation Review, vol. 10, n. 2, pp. 99-123.

GILI C. (2005), La credibilità. Quando e perché la comunicazione ha successo, Rubbettino, Soneria Mannelli, Catanzaro.

HELM S. (2005), "Designing a Formative Measure for Corporate Reputation", Corporate Reputation Review, vol. 8, n. 2, pp. 95-109.

HSIAO C. (2003), Analysis of panel data, Cambridge University Press, Cambridge.

INVERNIZZI E., ROMENTI S. (2005), "La valutazione dei risultati della Corporate communication”, Sinergie, n. 68, pp. 41-54. 
MAZZEI A., GAMBETTI R. (2006), "La comunicazione aziendale a sostegno del corporate branding e della reputazione: la gestione integrata di segni, comportamenti e reti di relazioni”, Atti del Convegno "Le tendenze del Marketing, Università Ca' Foscari Venezia, 20-21 gennaio.

MILGROM P.R., ROBERT J.W. (1982), "Distributional Strategies for Games with Incomplete Information", Mathematics of Operations Research, vol. 10, pp. 619-631.

MOLTEMI L., TROILO G. (2003), Ricerche di marketing, McGraw-Hill, Milano.

MUTTI A. (2007), "Reputazione", Rassegna Italiana di Sociologia, n. 4, pp. 601-622.

NELLI R.P., BENSI P. (2003), L'impresa e la sua reputazione. L'evoluzione della media coverage analysis, Vita \& Pensiero, Milano.

OTUBANJO O., AMUJO O.C., CORNELIUS N. (2010), "The Informal Corporate Identity Communication Process", Corporate Reputation Review, vol. 13, n. 3, pp. 157-171.

PASTORE A., VERNUCCIO M. (2008), Impresa e comunicazione. Principi e strumenti per il management, Apogeo, Milano.

PRUZAN P. (2001), "Corporate Reputation: Image and Identity", Corporate Reputation Review, vol. 4, n. 1, pp. 50-64.

RAVASI D., GABBIONETA C. (2004), "Le componenti della reputazione aziendale. Indicazioni dalla ricerca RQ Italy", Economia \& Management, n. 3, pp. 77-100.

RINDOVA V.P., WILLIAMSON I.O., PETKOVA A.P., SEVER J.M. (2005), "Being good or being known: An empirical examination of the dimensions, antecedents, and consequences of organizational reputation", Academy of Management Journal, vol. 48, n. 6, pp. 1033-1049.

ROBERTS P.W., DOWLING G.R. (2002), "Corporate Reputation and sustained superior financial performance”, Strategic Management Journal, vol. 23, n. 12, pp. 1077-1093.

ROMENTI S. (2008), "Corporate Governance e reputazione: dallo stakeholder relationship management allo stakeholder engagement", Impresa Progetto, n. 2, pp. 1-23.

SIANO A. (2001), Competenze e comunicazione del sistema d'impresa. Il vantaggio competitivo tra ambiguità e trasparenza, Giuffrè, Milano.

WALKER K. (2010), "A Systematic review of the Corporate Reputation Literature: Definition, Measurement, and Theory", Corporate Reputation Review, vol. 12, n. 4, pp. 357-387.

WALSH G., MITCHELL V.W., JACKSON P.R., BEATTY S.E. (2009), "Examining the Antecedents and consequences of Corporate Reputation: A Customer Perspective", British Journal of Management, vol. 20, n. 2, pp. 187-203.

WINN M.I., MACDONALD P., ZIETSMA C. (2008), "Managing Industry Reputation: The Dynamic Tension Between Collective And Competitive Reputation Management Strategies", Corporate Reputation Review, vol. 11, n. 1, pp. 35-55. 
